\title{
AI-Powered Hand-held Citrus Disease \& Fruit Spoilage Detection System
}

\author{
Chrissy Elizabeth Samuel ${ }^{1}$ Ahalya Chadran ${ }^{2}$, Anjali Krishna' Kavya V B ${ }^{4}$ \\ ${ }^{1} \mathrm{UG}$ - Student, Food Technology, Kerala University of Fisheries and Ocean Studies, Kerala \\ ${ }^{2}$ UG -Student, Food Technology, Kerala University of Fisheries and Ocean Studies, Kerala \\ ${ }^{3} \mathrm{UG}$ - Student, Food Technology, Kerala University of Fisheries and Ocean Studies, Kerala \\ ${ }^{4} \mathrm{UG}$ - Student, Food Technology, Kerala University of Fisheries and Ocean Studies, Kerala
}

\begin{abstract}
In food and agricultural technology, the use of image processing techniques has excellent indications and associations. These visual images are a significant source of information. Fruit classification has become one of the important applications which can be utilized not only in the supermarkets and grocery stores but also for the agriculturist to detect the diseases and work out different methods to ensure that these diseases did not occur in the next harvest. In order to address these identified problems, fruit classification and recognition of these diseases. We identified different methods which are commonly used in addressing vegetable and fruit classification and in recognizing diseases. And we used that surveyed image processing techniques for fruit disease detection, segmentation, and classification. The methods utilized in my project are able to distinguish among different types of citrus fruits and their diseases that are very alike in colour and texture.

With the expansion in technology, there's an increasing demand for solving lifestyle tasks. We know that refrigeration is the most commonly used technique for the storage of food, which works by lowering the reproduction rate of bacteria found in the food. Through this project, we are presenting the likelihood of solving the fruit spoilage problem, with technical assistance to spot the spoilage by continuous sensing. We expand a way to detect spoilage for fruits using sensor MQ135 sensor related to Arduino. The results of the findings are shown to support the freshness and quality of the fruit. the knowledge regarding the fruit spoilage is going to be conveyed to the purchasers using visual media by displaying the result to the user on the LCD screen
\end{abstract}

Keywords: Detection, Classification, MQ135 Sensor, Arduino

\section{INTRODUCTION}

The present method for fruit disease detection is naked eye observation, which is the crude method for observation. For further analysis of disease laboratory equipment and sophisticated devices are used. If the disease detection is not done appropriately it may lead to the addition of irrelevant pesticides that can cause long-term resistance and reduce the ability of the plant to fight back. We adopt AI image processing using Raspberry Pi hardware to detect citrus fruit diseases. This concept is low-cost and reliable method to verify if the fruit is fit for consumption or not. It is handy and portable and can be used by anyone like the common farmers, agriculturists, pomologists, etc. to detect the citrus fruit disease that affects the fruit. Hence, we are proposing a fruit disease detection project mainly based on the CNN strategy. It is a deep neural network most commonly applied to analyze visual imagery. Here we are running a convolution neural network on Raspberry Pi and the image is captured using a Raspberry Pi camera.

The atmospheric factors like humidity and temperature were checked with systems like refrigeration, vacuum storage, etc., hence quality monitoring tools or plans are required in the groceries or industries in order to minimize the wastage as well as to ensure appropriate ambient requirements during the transportation and storage. After harvesting fruits release gases. In some cases, these gases are more or less toxic. Gas sensors are electronic devices that detect gases and measure their concentration. Sensors help in detecting food spoilage and reduce wastage of food for consumers. Here we are using MQ 135 sensor which is an air quality sensor. We will connect the sensor to Arduino then it will sense the gases, and we will get the air quality 
Vol. 8, Issue 8, August 2021

\section{DOI: 10.17148/IARJSET.2021.8815}

\section{LITERATURE REVIEW}

\section{Citrus fruit disease detection:}

In paper [1], 'Plant Disease Detection using CNN \& Remedy', the proposed system helps in the identification of plant disease and provides remedies that can be used as a defense mechanism against the disease. The database obtained from the Internet is properly segregated and the different plant species are identified and are renamed to form a proper database then obtain a test database which consists of various plant diseases that are used for checking the accuracy and confidence level of the project.

Then using training data trained their classifier and then output is predicted with optimum accuracy. They use Convolution Neural Network (CNN) which comprises different layers which are used for prediction

In paper [2] "Adapted Approach for Fruit Disease Identification using Images", an adaptive approach for the identification of fruit diseases is proposed and experimentally validated. The image processing based proposed approach is composed of the following main steps; in the first step K-Means clustering technique is used for the defect segmentation, in the second step some state-of-the-art features are extracted from the segmented image, and finally, images are classified into one of the classes by using a Multi-class Support Vector Machine.

Paper [10]- "Factors influencing the use of deep learning for plant disease recognition", This article provides an indepth analysis of the main factors that affect the performance of deep learning-based tools for plant disease recognition under realistic conditions. This indicates that, currently, tools for automatic recognition of plant diseases, rather than offering a definite answer, can at most provide a very educated guess that will allow its users to take some action promptly, especially when specialized technical assistance is not available.

The hardware proposed by all these papers is not handy and expensive. So, we thought to bring a handy device which is cheap and reliable. The budget for all the models is quite high for common farmers. All those experiments they have done in leaves and fruits so here we are focusing mainly on citrus fruits and also, they are focusing on the classification of fruits. In the developing countries experts are required to identify the disease which is time cumbersome and immoderate. We are following the technical aspects mentioned in the papers but we are trying to make it economical and useful to common people

\section{Fruit spoilage detection system:}

In paper [5]' smart stale food detector using IOT (Internet of Things)', it deals with the technologies along with internet of things using Arduino which employs the script programming and sensors like DHT sensor, moisture sensor, MQ3 Sensor, Arduino UNO etc. In this paper, we develop a food quality sensing/detecting technique. The sensors will be associated with Arduino.

In paper [6], "IoT based project for food quality and monitoring", the author proposed a device called Smart Plate consisting of a variety of sensors that are activated depending on the food item. This plate can be placed in any utensil and a panel can be used to select the type of food item.

Most of the programs used for the Arduino based sensors is written in java script. Java script does not have any multi processors or multi reading features and cannot be used for networking purposes as there is not sufficient assistance available. In our model we are using Arduino embedded C. Instead of MQ3 used in some models, we are selected MQ135 because of its efficacy and detection of air quality. MQ3 mainly used for alcohol ethanol and smoke detection purpose. But we are focusing on spoilage so we selected MQ135

\section{EXPERIMENTAL METHODS OR METHODOLOGY}

\section{Proposed System}

In the proposed system we use the $\mathrm{CNN}$ algorithm for detecting citrus fruit disease so that it will allow us to obtain maximum accuracy if the dataset is good. We collect the image using the Raspberry Pi camera module, then process 


\section{International Advanced Research Journal in Science, Engineering and Technology}

Vol. 8, Issue 8, August 2021

DOI: 10.17148/IARJSET.2021.8815

and run it using the Python computer language and the Open CV image processing library to determine whether the fruit is diseased or not, and predict the disease.

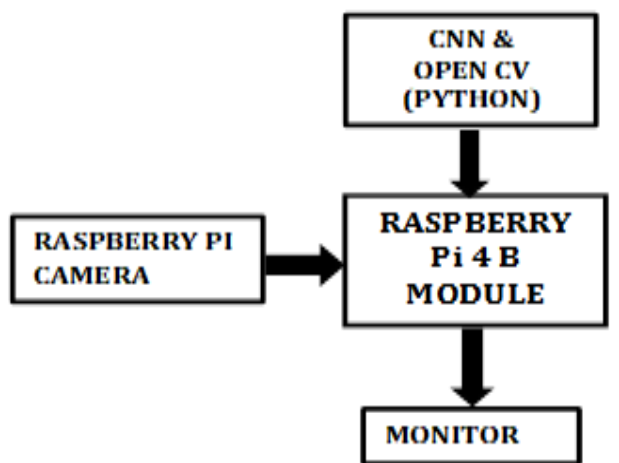

Fig 1. Citrus Fruit Disease Detection: Block Diagram

\section{Proposed algorithm}

- $\quad$ Collecting the Dataset- We need large amount of data to train our system, so that our model can learn from them by identifying out certain relations and common features related to the objects. This will help in training and testing our classifier. The dataset is created by downloading the images from Kaggle

\section{- $\quad$ Building the CNN}

The database has been pre-processed, including image resizing, reshaping, and array conversion. Our image dataset is used to train the system. When the camera captures a fruit image, the system uses a Convolutional Neural Network (CNN) algorithm to identify the fruit. Test images have also undergone similar processing. Feature extraction and classification are two characteristics of CNN.

Feature extraction: this is the process where the CNN will work a range of operations like the convolution and pooling . In this the CNN will distinguish and recognises the features.

Classification: Dependent or similar layers will assist as classifier. In our system the diseases are classifies in the order Class 1-Black spot, Class 2- Canker, Class 3- Greening, Class 4- Healthy, Class 5- Scab

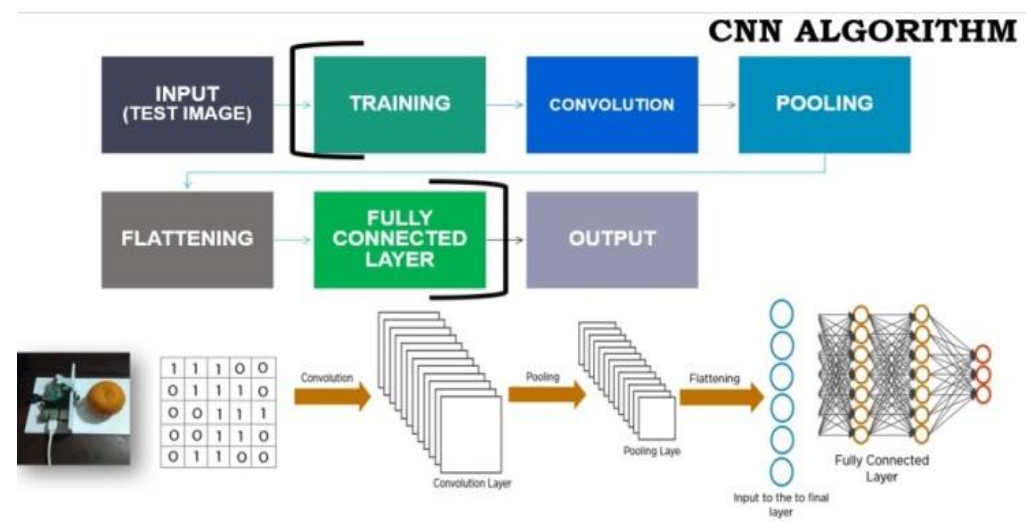

Fig 2. CNN Algorithm

- Data Augmentation

The data augmentation technique is used to enhance the number of images in a dataset by a large amount. We execute operations such as shift, rotation, zoom, and flip on the image dataset. Providing augmented photos to a model allows it 


\section{DOI: $10.17148 / I A R J S E T .2021 .8815$}

to learn characteristics from various sections of the same image more quickly, allowing it to perform better on unseen image data.

Image of citrus fruit image is captured and identified using CNN algorithm. The collected and identified image is then analysed for disease detection in the next stage. One of the most significant operations is the normalization of image size and format. Hereby using the Open CV framework and CNN algorithm the images are converted in some pixel size and some dots per inch

- $\quad$ Storage: This output is stored in python H5py file

\section{Fruits Spoilage Detection System using Arduino}

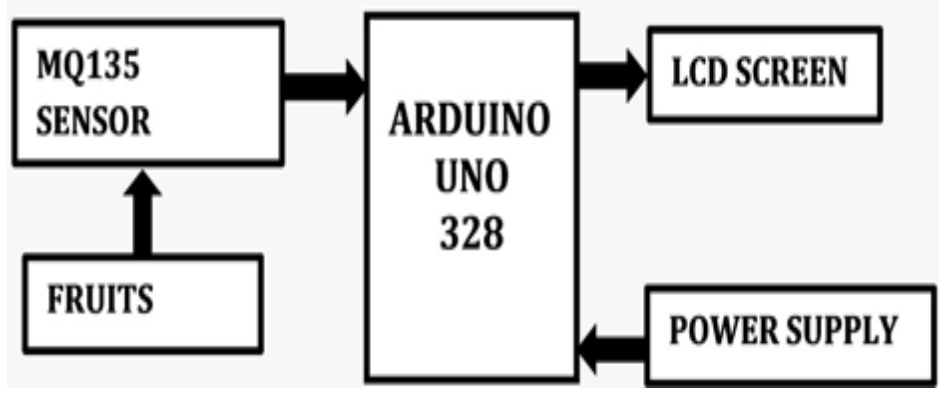

Fig 3. Fruits Spoilage Detection using Arduino: Block Diagram

In this system the gadget comprises of microcontroller Arduino UNO, MQ135 sensor and an LCD screen. MQ135 sensor detects the air quality changes and the microcontroller choose the results with the predefined calculation. The outcome is as 'fit for consumption' and not great to consume based on the sustenance freshness level

\section{RESULTS AND DISCUSSION}

Citrus Fruit Disease Detection System

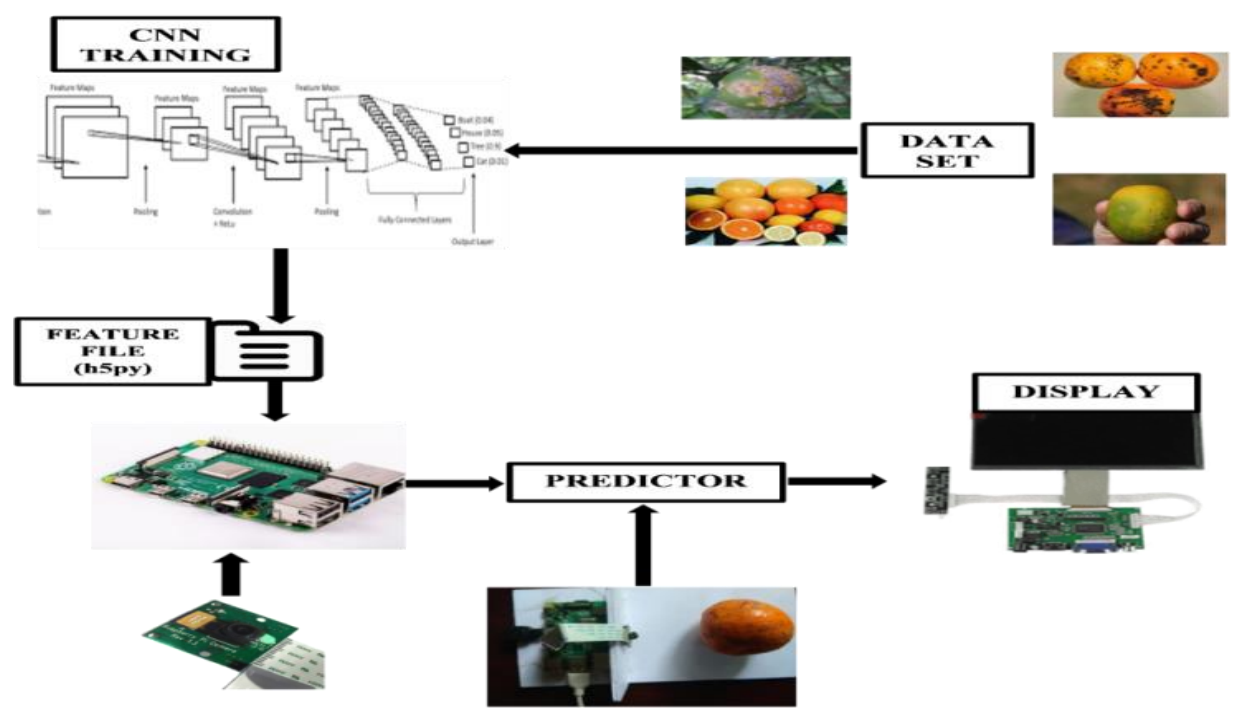

Fig 4. Experimental Setup for Citrus disease detection

The healthy and diseased citrus (for demonstration we have taken only two diseased ones greening and canker) fruits are taken and placed in front of the raspberry camera module as shown in (fig 5) then it is uploaded to the system and makes a comparison between the test image and the trained model, which is followed by the display of the disease. So, if there is any defect or disease in the fruit the software will displays it on the monitor like in the (fig6). 


\section{DOI: 10.17148/IARJSET.2021.8815}
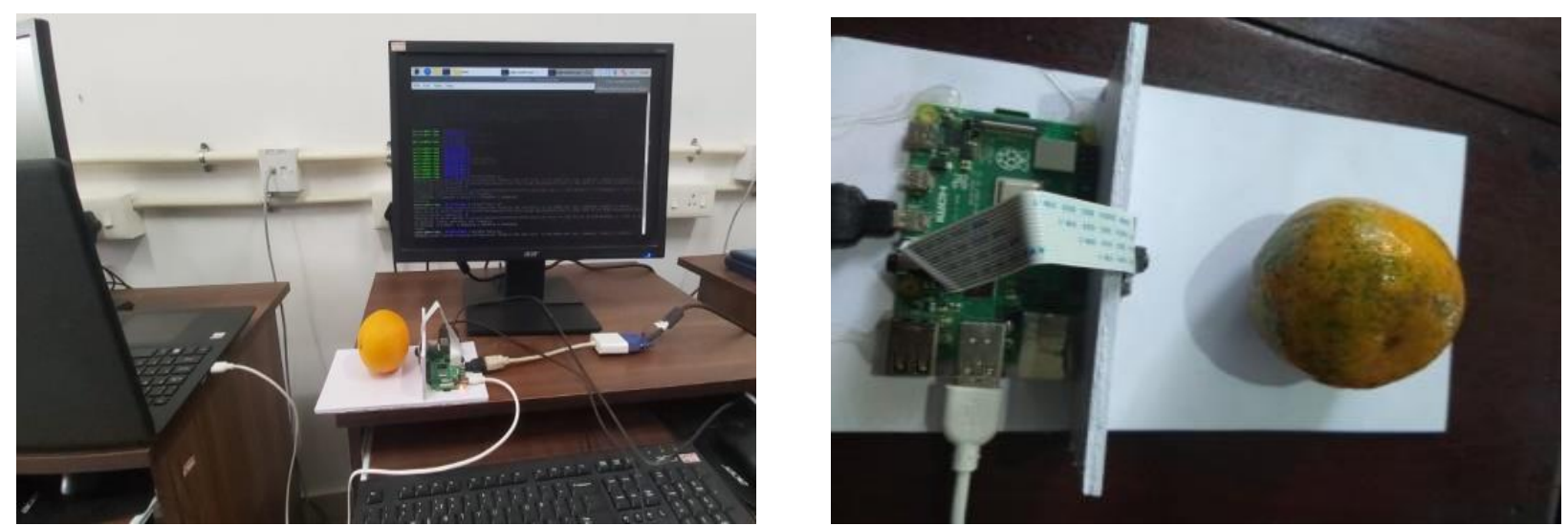

Fig 5. Raspberry Pi camera capturing image of diseased citrus(i)Canker

(ii) Greening

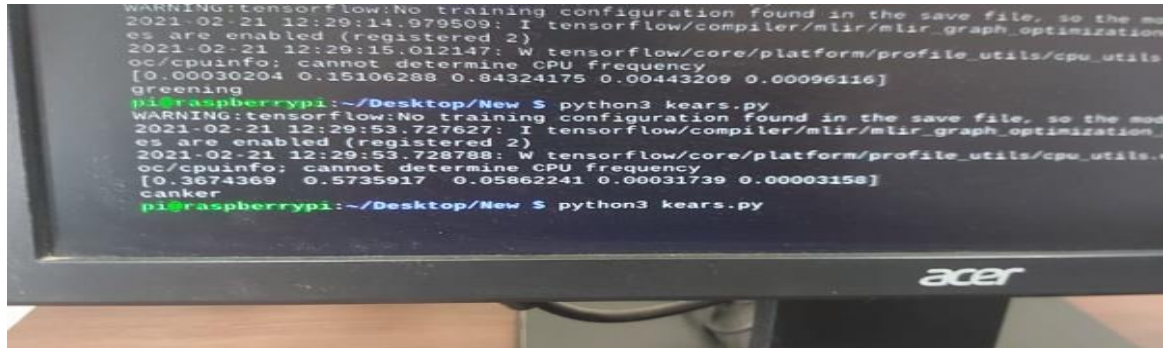

Fig 6. Displaying results for diseased citrus fruit (i) Greening (ii) Canker

\section{FRUITS SPOILAGE DETECTION-MQ135 ANALOG SERIAL VALUES}

In this session we will be discussing about how the Arduino based MQ135 sensor has able to sense the air quality change and there by detecting the degree of spoilage of three types of fruits. As the Arduino based MQ135 sensor is highly sensitive even low amount of air quality change in an air tight container can be identified which is generated by the decayed fruits. The levels of gas emission for three types of fruits have identified, and below we will present the table and the graphs for the experiments taken.

\section{Mango}
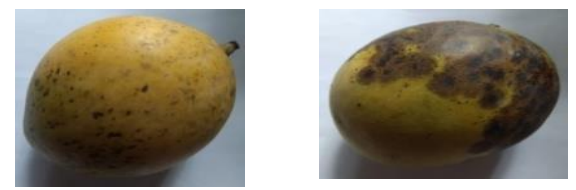

Fig 7. Fresh mango and spoiled mango taken as sample for experiment

\section{Tomato}
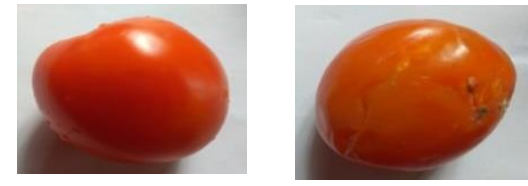

Fig 8. Fresh Tomato and spoiled tomato taken as sample for experiment

From the experiments that we carried out we observed the MQ135 Analog serial value range of fresh and spoiled Mango and Tomato at time intervals 5, $15 \& 30$ minutes respectively. Hence, we arrive at the inferred average ranges of 
Vol. 8, Issue 8, August 2021

DOI: 10.17148/IARJSET.2021.8815

fresh and spoiled Mango and Tomato as shown in the above Fig 9 and Fig 10.

\begin{tabular}{|c|c|c|c|c|c|c|c|c|c|c|}
\hline & \multicolumn{3}{|c|}{ READING 1} & \multicolumn{3}{|c|}{ READING 2} & & \multicolumn{3}{|c|}{ READING 1} \\
\hline & 5 min & $15 \min$ & $30 \mathrm{~min}$ & $5 \min$ & $15 \mathrm{~min}$ & $30 \mathrm{~min}$ & & $5 \min$ & $15 \mathrm{~min}$ & $30 \mathrm{~min}$ \\
\hline & 153 & 187 & 188 & 153 & 189 & 188 & & 252 & 288 & 333 \\
\hline & 154 & 190 & 190 & 154 & 190 & 190 & & 252 & 288 & 334 \\
\hline & 153 & 190 & 190 & 154 & 190 & 192 & & 253 & 288 & 333 \\
\hline & 155 & 192 & 192 & 155 & 192 & 193 & & 252 & 289 & 333 \\
\hline DAY & 154 & 193 & 193 & 154 & 193 & 195 & DAY & 252 & 288 & 334 \\
\hline 1 & 155 & 186 & 195 & 155 & 186 & 196 & 2 & 253 & 289 & 333 \\
\hline & 155 & 196 & 196 & 154 & 196 & 197 & & 253 & 288 & 334 \\
\hline & 155 & 198 & 198 & 155 & 198 & 197 & & 253 & 289 & 334 \\
\hline & 154 & 199 & 199 & 155 & 199 & 199 & & 253 & 288 & 334 \\
\hline & 155 & 199 & 199 & 155 & 199 & 199 & & 253 & 289 & 334 \\
\hline \begin{tabular}{|l} 
AVG \\
RNNGB
\end{tabular} & \begin{tabular}{|l|l|}
153.155 \\
\end{tabular} & 186.199 & 188.199 & 153.155 & $186-199$ & 188.199 & & 252.253 & 288.289 & 333-334 \\
\hline
\end{tabular}

\begin{tabular}{|c|c|c|c|c|c|c|c|c|c|c|}
\hline & \multicolumn{3}{|c|}{ READING 1} & \multicolumn{3}{|c|}{ READING 2} & & \multicolumn{3}{|c|}{ READING 1} \\
\hline & $5 \mathrm{~min}$ & $15 \mathrm{~min}$ & $30 \mathrm{~min}$ & $5 \mathrm{~min}$ & $15 \mathrm{~min}$ & $30 \mathrm{~min}$ & & $5 \mathrm{~min}$ & $15 \mathrm{~min}$ & $30 \mathrm{~min}$ \\
\hline & 100 & 97 & 96 & 105 & 95 & 94 & & 116 & 132 & 143 \\
\hline & 100 & 97 & 95 & 105 & 95 & 94 & & 117 & 132 & 143 \\
\hline DAY & 101 & 97 & 95 & 105 & 95 & 93 & DAY & 116 & 131 & 142 \\
\hline 1 & 98 & 96 & 95 & 105 & 94 & 94 & 2 & 117 & 132 & 143 \\
\hline & 99 & 95 & 95 & 104 & 95 & 94 & & 117 & 132 & 143 \\
\hline & 100 & 95 & 94 & 105 & 94 & 95 & & 116 & 131 & 143 \\
\hline & 99 & 95 & 95 & 104 & 94 & 94 & & 117 & 131 & 142 \\
\hline & 100 & 94 & 94 & 103 & 95 & 96 & & 117 & 132 & 141 \\
\hline & 100 & 95 & 95 & 105 & 95 & 93 & & 117 & 131 & 142 \\
\hline & 100 & 94 & 94 & 104 & 94 & 93 & & 117 & 131 & 140 \\
\hline $\begin{array}{l}\text { LNG } \\
\text { RANGE }\end{array}$ & 98.101 & 94.97 & 94.96 & 103.105 & 94.95 & 93.96 & & $116 \cdot 117$ & $131 \cdot 132$ & $140-143$ \\
\hline
\end{tabular}

Fig 9. MQ135 Analog serial values for Fresh Mango and Spoiled mango

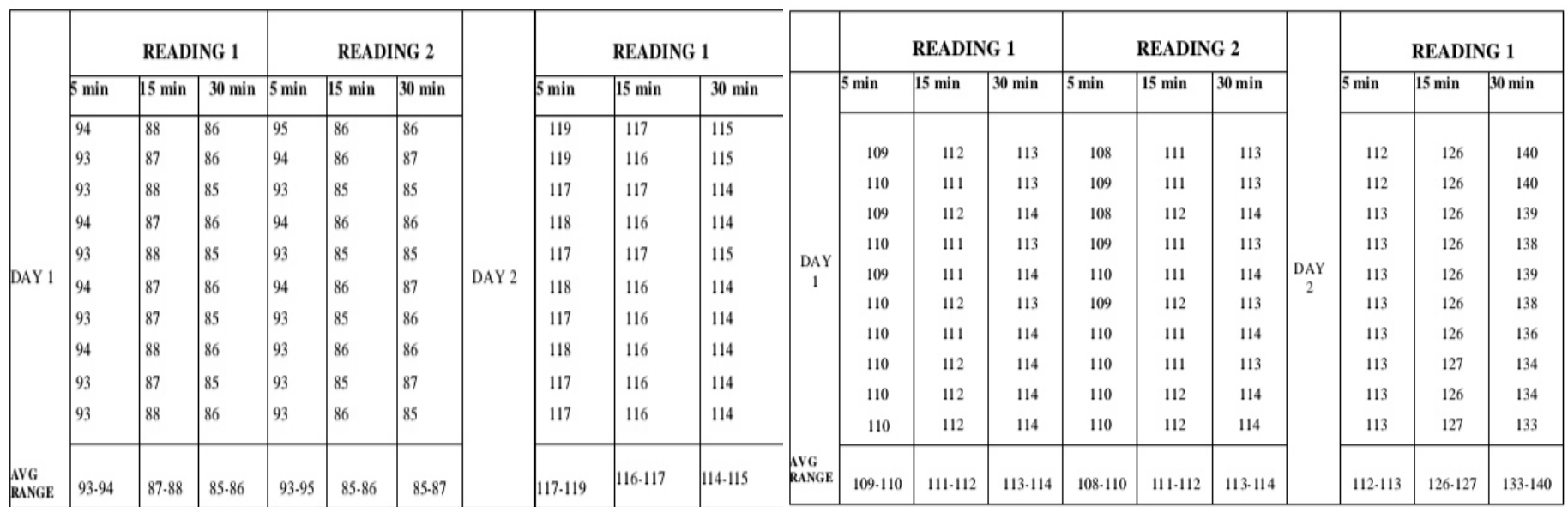

Fig 10. MQ135 Analog serial values for Fresh Tomato and Spoiled Tomato

\section{CONCLUSION}

In the present scenario accurate detection of disease and spoilage in fruit is gaining more importance for the successful crop cultivation. This can be carried out by digital image processing. Our motive is to develop a system that can detect fruit disease and its spoilage. So in our project, we adopt AI image processing technology using Raspberry Pi for disease detection.

Raspberry Pi improves the accuracy and speed of detection and classification of fruit disease. Here we are running a convolution neural network on Raspberry $\mathrm{Pi}$ and the image is captured using a Raspberry Pi camera. Also, this proposed system is able to solve the fruit spoilage problem with the help of Arduino and sensors. The network is trained in a systematic manner where we fruit images as input to the network and fruit diseases as the output of the network. After the training, the CNN model can predict disease of the fruit. We proposed a gadget based on Arduino UNO which is a well-known prototyping board. We can identify spoilage by continuous sensing. So for the spoilage detection system, we use an MQ135 sensor and Arduino boards. MQ 135 sensor is used for detecting the air quality. Our system will easily detect the freshness of any edible fruit and also a person consuming this fruit is aware of the quality before usage. Apart from that common farmer can use this portable device to detect the disease that affects the fruit. Our idea is cheap and reliable moreover the system is handy and can be used by anyone to make sure their fruit is spoiled 


\section{International Advanced Research Journal in Science, Engineering and Technology}

Vol. 8, Issue 8, August 2021

\section{DOI: $10.17148 /$ IARJSET.2021.8815}

\section{REFERENCES}

[1] Adnan Mushtaq Ali Karol , DrushtiGulhane, TejalChandiwade, 'Plant Disease Detection using CNN \&Remedy',International Journal of Advanced Research in Electrical, Electronics and Instrumentation Engineering ISSN (Print) : 2320 - 3765 Vol. 8, Issue 3, March 2019

[2] Shiv Ram Dubey, Anand Singh Jalal, 'Adapted Approach for Fruit Disease Identification using Images', International Journal of Computer Vision and Image Processing 2(3):51-65, July 2012

[3] Shivani and Sharanjith Singh'Fruit Disease Detection Using Convolution Neural Network Approach',Asian Journal of Computer Science and Technology ISSN: 2249-0701 Volume: 7 Issue: 5, 2018,pp-62-65

[4] Volker Dworak, JoernSelbeck , Karl-Heinz Dammer, Matthias Hoffmann, Ali Akbar Zarezadeh and Christophe Bobda, 'Strategy for the Development of a Smart NDVI Camera System for Outdoor Plant Detection and Agricultural Embedded System', Sensors ISSN 1424-8220 January 2013

[5] Sulakshana A. Gaikwad, Kalyani S.Deore, Monali K. Waykar,' Fruit Disease Detection and Classification', International Research Journal of Engineering and Technology -ISSN: 2395-0056 Volume: 04 Issue: 12, Dec-2017

[6] G. Sharada, S. Sindhoora, D. Sai Lakshmi Devi , Ms.PR Anisha4, Dr.BV Ramana Murthy \& Mr. C Kishor Kumar Reddy, 'Smart Stale Food detector using IoT (Internet of Things)', Global Journal of Engineering Science and Researches ISSN 2348 - 8034, 2019

[7] Sumathi MS, Thejaswini S, Pranav Kashyap, Shahinaanjum, Shashi Shanker, Shreya GK "IoT Based Project For Food Quality And Monitoring" International Journal On Recent And Innovation Trends In Computing And Communication ISSN: 2321-8169 Volume: 3 Issue: 5 3172 - 3174 January 2019

[8] Fatima Mustafa And Silvana Andreescu, "Chemical And Biological Sensors For Food-quality Monitoring And Smart Packaging”, Europe PMC, October 2018*Department Of Chemistry And Biomolecular Science, Clarkson University, Potsdam, NY 13699 USA Chemical And Biological Sensors For Food-quality Monitoring And Smart Packaging

[9] Samaneh, Matindoust, Majidbaghei-nejad, Mohammed Hadishahrokh Abadi, Zhouzou, Li-rongzheng, "Food Quality And Safety Monitoring Using Gas Sensor Array In Intelligent Packaging".

[10] G. Rama Mohan Reddy, Nettam Sai Sumanth, N. Sai Preetham Kumar , 'Plant Leaf Disease Detection Using Cnn And Raspberry Pi' , International Journal Of Advance Scientific Research And Engineering Trends, Volume 5 , Issue 2 , February 2020

[11] R Chandramma, Ramya C, S Vinitha, Shivani P R, Vidya M S, 'Automatic Fruit and Vegetable Detection and Disease Identification System', International Journal of Engineering Research \& Technology ISSN: 2278-0181, 2020

[12] Mandeep Singh Centre For Development Of Advanced Computing, 'Hand Held Device For Detection Of Pesticides Using NDVI', International Journal Of Computer Applications November 2016

[13] Jayme Garcia ArnalBarbedo,'Factors influencing the use of deep learning for plant disease recognition', Biosystems Engineering' August 2018

[14] Arun Kumar G,' An Arduino sensor-based approach for detecting the food spoilage', International Journal of Engineering Applied Sciences and Technology ISSN No. 2455-2143 Vol : 5, Issue: 3, 2019, Pages 596-599,2019

[15] Jamil Ahmad, Bilal Jan, Hakeem Farman, Wakeel Ahmad and Atta Ullah, Disease Detection in Plum Using Convolutional Neural Network under True Field Conditions, Sensors, MDPI, September 2020

[16] Sachin D. Khirade and A. B. Patil. "Plant Disease Detection Using Image Processing." International Conference on Computing Communication Control and Automation (ICCUBEA), 2015 International Conference on, pp. 768-771. IEEE, 2015

[17] Prakash T; Arun G; Arun Kumar B; Deepan S; Kalyana Venkata Ramanan. "IoT Based Smart Irrigation for Multi Cropping System". International Research Journal on Advanced Science Hub, 2, 4, 2020, 7-12. doi: 10.47392/irjash.2020.20

[18] Deshmukh K P. "Wireless Transceiver Module HC-12 based Automatic Water-level Monitoring and Control System". International Research Journal on Advanced Science Hub, 2, 10, 2020, 24-28. doi: 10.47392/irjash.2020.184

[19] Mr. Dinesh; Mr. Meiyarasan; Mr. Prabu. "Health Monitoring System Using IOT Sensor". International Research Journal on Advanced Science Hub, 3, 4, 2021, 83-89. doi: 10.47392/irjash.2021.119

[20] Mr. Jeelani; Kishan Pal Singh; Aasim Zafar. "A Trust Calculation Algorithm for Communicating Nodes in Wireless Sensor Networks". International Research Journal on Advanced Science Hub, 3, Special Issue ICARD-2021 3S, 2021, 145-152. doi: 10.47392/irjash.2021.083 